\title{
DETERMINAÇÃO DO PONTO DE AMOSTRAGEM PARA A QUANTIFICAÇÃ O DE MICRONUTRIENTES EM ACÁCIA-NEGRA (Acacia mearnsii De Wild.)
}

\author{
Fábio Luiz Fleig Saidelles ${ }^{1}$, Marcos Vinícius Winckler Caldeira ${ }^{2}$, Mauro Valdir Schumacher ${ }^{3}$, \\ Rafaelo Balbinot ${ }^{4}$
}

\begin{abstract}
${ }^{1}$ Eng. Florestal, Dr., FEPAGRO, Santa Maria, RS, Brasil - fabio-saidelles@ fepagro.rs.gov.br ${ }^{2}$ Eng. Florestal, Dr., Depto. de Engenharia Florestal, UNICENTRO, Irati, PR, Brasil - caldeiramv@yahoo.com.br

${ }^{3}$ Eng. Florestal, Dr., Depto. de Ciências Florestais, UFSM, Santa Maria, RS, Brasil - schumacher@ pesquisador.cnpq.br

${ }^{4}$ Eng, Florestal, M.Sc., Doutorando em Eng. Florestal, UFPR, Curitiba, PR, Brasil - rbalbinot@yahoo.com.br
\end{abstract}

Recebido para publicação: 26/09/2007 - Aceito para publicação: 04/08/2008

\begin{abstract}
Resumo
O objetivo deste estudo foi determinar o ponto ótimo de amostragem para a quantificação de nutrientes em árvores de Acacia mearnsii. O trabalho foi realizado em uma plantação com quatro anos de idade localizada na cidade de Arroio dos Ratos (RS), em uma fazenda pertencente à empresa Agroseta S.A., nas coordenadas $30^{\circ} 07^{\prime} 12^{\prime \prime}$ de latitude sul e $51^{\circ} 57^{\prime} 45^{\prime \prime}$ de longitude oeste, com altitude média de $90 \mathrm{~m}$. Após o inventário florestal, foram abatidas 21 árvores, distribuídas em 7 classes diamétricas, sendo determinados a biomassa e o teor de micronutrientes dos componentes folha, galho vivo, galho morto, raiz, madeira e casca. Para os componentes madeira e casca foram retiradas amostras ao longo do fuste nas posições de 1, 3, 10, 20, 30, 40, 50, 60, 70, 80 e $90 \%$ da altura total das árvores. Os teores dos micronutrientes $\mathrm{B}, \mathrm{Cu}, \mathrm{Fe}, \mathrm{Mn}$ e $\mathrm{Zn}$ não demonstraram variação significativa ao longo do fuste nos componentes madeira e casca, indicando que a amostragem para quantificação de nutrientes pode ser feita a qualquer altura. É possível a estimativa do estoque de micronutrientes por amostras coletadas no DAP aos 4 anos de idade. Recomenda-se para isso a utilização de trado, para não ser necessário derrubar as árvores amostradas.

Palavras-chave: Nutrição florestal; avaliação nutricional arbórea; nutrientes minerais.
\end{abstract}

\begin{abstract}
Determination sampling point for micronutrients quantification in Acacia mearnsii De Wild. The objective of this study was to quantify the biomass and to determine the optimal sampling point for nutrients quantification in Acacia mearnsii trees. The work was conducted in black wattle stand, whit four years old, located in Arroio dos Ratos city in a farm belonging to Agroseta S.A., having as coordinates $30^{\circ} 07^{\prime} 12^{\prime \prime}$ of south latitude and $51^{\circ} 57^{\prime} 45^{\prime \prime}$ of longitude west, with $90 \mathrm{~m}$ of average altitude. After the forest inventory 21 trees were felled, distributed in 7 diametric classes, to cover the stand heterogeneity. The biomass and the micronutrients amount in the leaves, live branches, dead branches, roots, wood and bark were determined. Samples of wood and bark were taken along the stem in the positions of: $1,3,10,20,30,40,50,60,70,80$ and $90 \%$ of the total height. The micronutrients levels for $\mathrm{B}, \mathrm{Cu}, \mathrm{Fe}, \mathrm{Mn}$ and $\mathrm{Zn}$ didn't show significant difference along the stem to wood and bark, indicating that there isn't an exact point for sampling along the stem to nutrients quantification. It's possible to estimate the stock of micronutrients by samples collected at DBH to 4 years of age. For this it's recommended to use the borer, for not to fell the sampled trees.

Keywords: Forest nutrition; arboreal nutrition evaluation; mineral nutrient.
\end{abstract}

\section{INTRODUÇÃO}

O primeiro plantio de acácia-negra no Rio Grande do Sul foi realizado em 1918, segundo Oliveira (1968). Os plantios comerciais tiveram início em 1930, com a importação de $30 \mathrm{~kg}$ de sementes da África do Sul, e, em 1941, iniciou-se a utilização comercial dessa espécie com a criação da Sociedade Extrativa de Tanino de Acácia Ltda. (SETA). Higa; Dedecek (1999) estimaram que a área plantada dessa espécie em 1999 era de aproximadamente 100.000 ha. 
Segundo Resende et al. (1992), da acácia-negra pode-se utilizar desde a madeira até a casca. Da casca, extrai-se o tanino, com utilização no curtimento de couro e peles, na produção de anticorrosivos e até de substâncias usadas no tratamento da água. Já a madeira pode ser usada como lenha ou carvão e é considerada excelente matéria-prima para a fabricação de celulose e papel de superior qualidade.

As plantações de acácia-negra têm características multifuncionais: agem na recuperação de solos de baixa fertilidade, permitem consórcio com cultivos agrícolas e criação de animais, e de suas árvores, além da madeira, é possível o uso da casca para fins industriais (EMBRAPA, 2005). Carpanezzi (1998) destaca a acácia-negra como espécie ideal na recuperação ambiental, pois é uma espécie pioneira de vida curta que cobre rapidamente o solo, não é invasora, não apresenta rebrota de cepa, não inibe a sucessão local e enriquece o solo pela elevada deposição de folhedo rico em nitrogênio.

Segundo Schneider et al. (2000), a importância da espécie está relacionada ao fato de ela permitir ser cultivada pelos pequenos agricultores em sistemas agrossilvipastoris. No início do desenvolvimento, quando as plantas apresentam um dossel aberto, elas possibilitam que os produtores cultivem milho, melancia e outras culturas em consórcio com a acácia-negra. Mais tarde, quando o dossel está fechado, a floresta proporciona consórcio com a pecuária, aumentando com isso a rentabilidade do investimento.

O conhecimento sobre o teor, bem como sobre a distribuição dos nutrientes nas árvores, é fundamental, segundo Caldeira (1998), para estabelecer estratégias de amostragem com a finalidade de estudar a nutrição, ciclagem e exportação. Conforme Kimmins (1987), para manter a produtividade em qualquer sítio, é necessário determinar a duração do ciclo ecológico, o período necessário para que o sítio recupere as condições antes da exploração, ou uma adequada adubação. Isso só será possível através da quantificação do conteúdo de nutrientes no sítio, nas exportações através de colheita, fogo e erosão e na reposição natural, ou seja, deposições atmosféricas, mineralização e fixação biológica de nitrogênio.

A determinação do teor de nutrientes nos componentes das árvores, segundo Montagnini e Sancho (1994), e a determinação de biomassa podem ser utilizados como indicadores dos impactos ambientais potenciais das árvores e da colheita florestal sobre os nutrientes no sítio, permitindo, assim, segundo Drumond et al. (1997), adoção de estratégias de manejo que possam levar à redução da perda de nutrientes.

A determinação do teor de nutrientes nas diversas partes do vegetal permite calcular a mineralomassa em função da fitomassa estimada, e o monitoramento da decomposição da serapilheira fornece dados essenciais sobre a liberação de nutrientes para o solo da floresta (O'CONNELL; SANKARAN, 1997).

O conhecimento dos teores dos nutrientes ao longo do fuste das árvores é importante para que se possa determinar um ponto exato para a retirada das amostras, o que facilitará a determinação das quantidades armazenadas na madeira e na casca (HOPPE, 2003). Apesar disso, é importante ressaltar a raridade de trabalhos sobre o conhecimento dos teores dos nutrientes ao longo do fuste das árvores. Isso se deve principalmente à dificuldade na coleta de dados, especialmente em florestas nativas, devido à complexidade dessas formações, dos custos elevados para a realização de estudos, do grande número de pessoas necessárias e da falta de metodologia adequada.

Em função do rápido crescimento da espécie e da demanda pela madeira na região, que tem reduzido o tempo de corte, é importante a análise de nutrientes da árvore nesse ciclo curto de rotação mais crítico para o sítio. Portanto, o presente trabalho, realizado em povoamentos de Acacia mearnsii com quatro anos de idade estabelecido no Rio Grande do Sul, teve como objetivo determinar a altura de amostragem dos componentes (madeira e casca) das árvores para a determinação dos micronutrientes.

\section{MATERIAL E MÉTODOS}

\section{Características da área de estudo}

Este estudo foi realizado na Fazenda Chagastelles, pertencente à empresa Agroseta S.A., distante aproximadamente $4 \mathrm{~km}$ da cidade de Arroio dos Ratos (RS). Distante cerca de $60 \mathrm{~km}$ de Porto Alegre, o município de Arroio dos Ratos localiza-se na depressão central do estado do Rio Grande Sul, na microrregião do Vale do Jacuí, limitada ao norte pela encosta do planalto basáltico e ao sul pelo escudo rio-grandense, situada entre as coordenadas geográficas $30^{\circ} 07^{\prime} 12^{\prime \prime}$ de latitude sul e $51^{\circ} 57^{\prime} 45^{\prime \prime}$ de longitude oeste, com altitude média de $90 \mathrm{~m}$. 
De acordo com a classificação de Köppen, o clima da região é do tipo Cfa, subtropical (MORENO, 1961). A temperatura média do mês de janeiro fica em torno de $24^{\circ} \mathrm{C}$, a do mês de julho é de $13{ }^{\circ} \mathrm{C}$ e a temperatura média anual fica em torno de $18-19{ }^{\circ} \mathrm{C}$, sendo que a temperatura média das máximas no ano é de $24{ }^{\circ} \mathrm{C}$ e a das mínimas é de $14^{\circ} \mathrm{C}$. A precipitação pluvial no ano varia de 1200 a $1600 \mathrm{~mm}$ e é bem distribuída, ocorrendo de 100 a 120 dias de chuva no ano. A região de Arroio dos Ratos possui, de maio a agosto, $600 \mathrm{~h}$ de frio, abaixo de $10^{\circ} \mathrm{C}$, e $200 \mathrm{~h}$ de frio, de maio a agosto, abaixo de $7{ }^{\circ} \mathrm{C}$. A umidade relativa do ar média, a radiação solar média e a insolação anual total variam, respectivamente, de 75 a $80 \%$, de 325 a 375 cal.cm $^{-2} \cdot$ dia $^{-1}$ e de 2200 a 2600 h (INSTITUTO DE PESQUISAS AGRONÔMICAS, 1989). O solo da área de estudo (Tabela 1), segundo a Embrapa (2006), é classificado como Argissolo Vermelho distrófico típico, localizado em relevo suave ondulado.

Tabela 1. Análise química do solo plantado com Acacia mearnsii com quatro anos de idade.

Table 1. Chemical analysis of soil planted with Acacia mearnsii with four years old.

\begin{tabular}{|c|c|c|c|c|c|c|c|c|c|c|c|c|c|c|}
\hline \multirow{2}{*}{$\begin{array}{l}\text { Prof. } \\
\text { cm }\end{array}$} & \multirow{2}{*}{$\begin{array}{l}\text { Argila } \\
\text { g.kg }^{-1}\end{array}$} & \multirow{2}{*}{$\begin{array}{r}\mathbf{p H} \\
\mathrm{H}_{2} \mathrm{O}\end{array}$} & M.O & $\mathbf{m}$ & V & \multirow{2}{*}{\multicolumn{2}{|c|}{$\begin{array}{l}\mathbf{P} \\
\text { mg. }^{-1}\end{array}$}} & $\mathbf{C a}$ & $\mathrm{Mg}$ & CTC & $\mathbf{C u}$ & $\mathrm{Zn}$ & $\mathrm{Fe}$ & Mn \\
\hline & & & & $\%$ & & & & \multicolumn{3}{|c|}{$\operatorname{cmol}_{\mathbf{c}} \cdot \mathrm{L}^{-1}$} & \multicolumn{4}{|c|}{ mg. $\mathrm{L}^{-1}$} \\
\hline $0-10$ & 310 & 4,4 & 2,5 & 27 & 44 & 4,0 & 86 & 2,0 & 0,5 & 4,8 & 1,8 & 1,6 & 123 & 90 \\
\hline $10-20$ & 350 & 4,3 & 2,0 & 27 & 41 & 3,2 & 112 & 1,5 & 0,5 & 3,9 & 1,9 & 1,3 & 133 & 76 \\
\hline $20-30$ & 440 & 4,4 & 1,8 & 30 & 39 & 3,2 & 114 & 2,2 & 0,8 & 4,7 & 2,3 & 0,8 & 64 & 38 \\
\hline $30-40$ & 460 & 4,4 & 1,8 & 41 & 35 & 3,2 & 78 & 2,1 & 0,9 & 4,9 & 2,2 & 0,5 & 54 & 6 \\
\hline $40-50$ & 430 & 4,4 & 1,7 & 33 & 40 & 3,2 & 78 & 1,8 & 0,8 & 4,7 & 2,0 & 0,4 & 51 & 6 \\
\hline $50-60$ & 560 & 4,4 & 1,6 & 35 & 40 & 2,5 & 90 & 1,9 & 0,9 & 5,0 & 1,9 & 0,4 & 45 & 1 \\
\hline
\end{tabular}

\section{Implantação do povoamento}

O plantio foi realizado em setembro de 1997, numa área de 2.564 ha, com espaçamento de $1,3 \mathrm{x}$ $3 \mathrm{~m}$, tendo como preparo de solo a subsolagem com uma haste, alcançando a profundidade de $45 \mathrm{~cm}$, e gradagem na linha de plantio. O plantio foi realizado com semente, utilizando a plantadeira hidráulica de acácia-negra (PHA). Em cada cova, foram colocadas oito sementes. Simultaneamente com o plantio, foi realizada a adubação, utilizando-se 40 gramas por cova de NPK (02-32-06). Outra etapa foi o replantio, realizado aproximadamente 30 dias após a implantação do povoamento.

Em relação aos tratos culturais, foi aplicado herbicida seletivo pré-emergente (Gamit $360 \mathrm{cs}$ ), na dose de $400 \mathrm{ml} \mathrm{ha}^{-1}$, e, em maio de 1998, foi realizado desbaste e limpeza da área, utilizando-se foice manual. Foi realizado ainda o combate à formiga cortadeira e, mais tarde, o controle do Oncideres inpluviata (serrador).

\section{Inventário florestal da área amostral}

Em uma área homogênea quanto às condições de sítio, onde foram demarcadas 7 parcelas de formato retangular de $20 \times 30 \mathrm{~m}\left(600 \mathrm{~m}^{2}\right)$, em cada uma das parcelas, foram medidos os diâmetros à altura do peito (DAP) de todas as árvores, com suta, e a altura (h) de 10\% das alturas dessas mesmas árvores, com aparelho Vertex.

\section{Determinação da biomassa acima do solo}

Após a determinação da distribuição diamétrica e sua divisão em classes de diâmetro, foram selecionadas três árvores em cada uma delas, de modo que a maior amplitude dos dados fosse contemplada. Selecionadas as árvores, de acordo com as classes de DAP, elas foram derrubadas e mensuradas quanto à altura total. Na sequência, os galhos foram separados do tronco e classificados em vivos (verdes) ou mortos (secos). Dos galhos verdes foram separadas todas as folhas. O tronco foi descascado, sendo separado em dois componentes: madeira e casca. Ainda no campo, os componentes (madeira, casca, folhas, galhos vivos e galhos mortos) tiveram seu peso úmido determinado, mediante pesagem com utilização de uma balança de gancho.

Logo após, foram retiradas amostras de todos os componentes das árvores, as quais eram pesadas em balança de precisão de $0,01 \mathrm{~g}$. Para a madeira e casca, foram retiradas amostras nas posições de 1,3 , $10,20,30,40,50,60,70,80$ e $90 \%$ da altura total das árvores.

Com o peso úmido determinado no campo, as amostras foram devidamente identificadas e encaminhadas para o Laboratório de Ecologia Florestal da Universidade Federal de Santa Maria, onde 
foram postas em estufa de circulação de ar, à temperatura de $65{ }^{\circ} \mathrm{C}$, até atingirem peso constante. Estabelecida a relação peso seco/úmido, foram calculados os pesos secos totais de cada componente de árvore e, com isso, foi determinada a biomassa seca $(\mathrm{kg})$.

\section{Determinação da biomassa abaixo do solo}

Todas as árvores amostradas, ou seja, 21, tiveram suas raízes escavadas com auxílio de um trator. Por meio do uso de bomba de pressão, foram submetidas a lavagem, na qual o solo aderido às raízes foi totalmente removido e todas as raízes com diâmetro acima de $0,50 \mathrm{~cm}$ foram coletadas. As amostras da raiz foram retiradas nesse momento, com a determinação do seu peso úmido, sendo posteriormente levadas ao laboratório, onde foi realizado o mesmo procedimento adotado para as outras amostras.

\section{Determinação do teor de nutrientes nos componentes das árvores}

No laboratório de Ecologia Florestal, as amostras secas foram moídas e submetidas às análises químicas para obtenção dos teores de nutrientes. Os micronutrientes determinados foram: boro (B), cobre $(\mathrm{Cu})$, ferro $(\mathrm{Fe})$, manganês $(\mathrm{Mn})$ e zinco $(\mathrm{Zn})$, com base na metodologia descrita por Tedesco et al. (1995) para análises de tecidos vegetais.

\section{Relação entre o DAP e o teor de micronutrientes}

Com os dados dos teores de micronutrientes $\left(\mathrm{mg} \mathrm{kg}^{-1}\right)$, obtidos na análise dos tecidos pelo produto com a biomassa de cada componente $(\mathrm{kg})$, estimou-se a quantidade de micronutrientes dos componentes $\left(\mathrm{mg}\right.$.árvore $\left.{ }^{-1}\right)$. A partir do DAP das árvores amostradas, foi usada uma equação $\left(\log \mathrm{y}=\mathrm{b}_{0}+\right.$ $\mathrm{b}_{1} \log$ DAP) para cada nutriente em cada componente das árvores, e, para cada equação, foram utilizados parâmetros estatísticos para averiguar se ela apresentava um bom ajuste aos dados, sendo eles o coeficiente de determinação ajustado $\left(\mathrm{R}_{\mathrm{aj}}^{2}\right)$ e o erro padrão da estimativa em percentagem $\left(\mathrm{S}_{\mathrm{yx}} \%\right)$. Só então as equações foram utilizadas para estimar os conteúdos dos nutrientes dos componentes das árvores de Acacia mearnsii do restante da parcela. As equações baseadas no DAP também foram utilizadas para estimar o teor de carbono orgânico das árvores.

\section{Quantidade de micronutrientes nos componentes da biomassa por hectare}

As quantidades de micronutrientes por hectare $\left(\mathrm{g} \cdot \mathrm{ha}^{-1}\right)$ foram determinadas com base na média do teor de cada micronutriente $\left(\mathrm{mg}^{\mathrm{kg}} \mathrm{kg}^{-1}\right)$ dos componentes das árvores pelo produto com a biomassa $\left(\mathrm{mg}_{\mathrm{g}} \mathrm{ha}^{-1}\right)$ dos respectivos componentes, considerando isso pelas classes diamétricas distribuídas no hectare, fazendo o somatório.

\section{Determinação do teor de micronutrientes ao longo do fuste para madeira e casca}

Foram coletadas amostras ao longo do fuste nas posições a 1, 3, 10, 20, 30, 40, 50, 60, 70, 80 e $90 \%$ da altura total das árvores. As amostras foram secas, moídas e analisadas, para determinação dos teores dos micronutrientes nesses compartimentos.

\section{Determinação do "teor representativo"}

$\mathrm{O}$ teor que representa o teor de nutrientes ao longo do fuste para madeira e casca, chamado de "teor representativo", serve para a determinação da quantidade de nutrientes no fuste.

Para o cálculo do teor representativo, que nada mais é do que o teor de nutriente ponderado em função da biomassa, utilizou-se a massa de nutriente de cada árvore, obtida pela multiplicação do teor de nutriente encontrado nas posições amostradas ao longo do fuste pelas respectivas biomassas dos componentes (madeira e casca), expressa em miligrama (mg) para os micronutrientes. Com o quociente da massa total de cada micronutriente e a biomassa total acumulada nos componentes (madeira e casca) das 21 árvores amostradas, obteve-se o teor representativo.

\section{Determinação da "altura representativa"}

O termo "altura representativa" expressa a altura no fuste da qual pode ser retirada uma amostra que represente a quantidade de nutrientes no fuste para os componentes (madeira e casca). Para determinar a altura representativa da amostragem para a quantificação dos nutrientes no fuste, para 
madeira e casca, deve-se, em primeiro lugar, observar qual o nutriente que apresentou variação ao longo do fuste e, depois, usar o teor representativo para determinar a altura ideal da amostragem.

\section{Análise estatística}

Com objetivo de determinar o teor dos micronutrientes nos componentes (madeira e casca) nas diferentes alturas amostradas, verificou-se, através do teste de Tukey ao nível de 5\%, se existe diferença significativa entre os teores encontrados ao longo do fuste.

\section{RESULTADOS E DISCUSSÃO}

\section{Inventário florestal}

As alturas das árvores que não foram medidas foram estimadas através da equação de relação hipsométrica (h/d), que apresentou os melhores parâmetros de ajuste para a região e para a espécie, na época da realização da pesquisa (Tabela 2).

Tabela 2. Modelo de regressão testado para estimar as alturas das árvores de Acacia mearnsii com quatro anos de idade.

Table 2. Tested regression model to estimate the heights of trees of Acacia mearnsii with four years old.

\begin{tabular}{|c|c|c|c|c|}
\hline \multirow{2}{*}{ Modelo } & \multicolumn{2}{|c|}{ Coeficientes } & \multirow{2}{*}{$\mathbf{R}_{\text {aj }}^{2}$} & \multirow{2}{*}{$\operatorname{Syx}(\%)$} \\
\hline & $\mathbf{b}_{0}$ & $b_{1}$ & & \\
\hline $\log \mathrm{h}=\mathrm{b}_{0}+\mathrm{b}_{1} * 1 / \mathrm{DAP}$ & 1,378574 & $-2,079563$ & 0,92 & 3,0 \\
\hline
\end{tabular}

H: altura (m); log: logaritmo de base 10; DAP:diâmetro à altura do peito $(\mathrm{cm})$.

Como se pode verificar, o baixo erro padrão e o alto coeficiente de determinação demonstrado pela equação resultou em um bom ajuste dos diâmetros com as alturas indicadas. Essa equação poderá ser usada para estimar as alturas das árvores de Acacia mearnsii com quatro anos.

Caldeira et al. (1998), trabalhando com procedências australianas de acácia-negra, aos 2,4 anos de idade, observou que o melhor modelo para Batemans Bay e Lake George Bunge Dore foi $h=1 /\left(b_{0}+\right.$ $\left.\left(b^{1} / \mathrm{DAP}\right)\right)^{2}+1,30$, com $\mathrm{R}_{\mathrm{aj}}^{2}=26,88$ e Syx $=1,55$ e $\mathrm{R}_{\mathrm{aj}}^{2}=45,13$ e Syx $=1,93$, respectivamente. No entanto, para Bodalla, o modelo escolhido foi $\log =b_{0}+b_{1} / \mathrm{DAP}, \operatorname{com~R}^{2}{ }_{\mathrm{aj}}=19,96$ e Syx $=5,09$. As três procedências apresentaram coeficiente de determinação ajustado baixo, o que pode ser atribuído ao fato do povoamento ser muito jovem, não tendo assim uma boa relação entre diâmetro e altura das árvores, bem como a outros fatores, como a qualidade do sítio. A Bodalla possui um crescimento maior em diâmetro do que em altura, que pode ser em função de ela possuir maiores percentagens de falhas. Pereira et al. (2000) trabalharam com povoamento comercial de acácia-negra aos 9 anos de idade. As alturas das árvores foram estimadas através do modelo $h=1 /\left(b_{0}+b_{1} / D_{A P}{ }^{2}\right)+1,30$, tendo um $F$ significativo e um $\mathrm{R}_{\mathrm{aj}}^{2}=83,4 \%$.

Com objetivo de selecionar modelos matemáticos para estimar a altura das árvores em função do DAP e da idade (2, 4, 6 e 8 anos), em povoamentos de acácia-negra (Acacia meanrsii De Wild.), na região sudeste do estado do Rio Grande do Sul, Caldeira et al. (2002) concluíram que a inclusão da variável idade no modelo matemático aumenta a precisão das estimativas de altura, quando se utiliza povoamentos com idades diferentes. Quando o objetivo é estimar a altura de povoamentos equiâneos, qualquer um dos cinco modelos matemáticos selecionados $\left(h=b_{o}+b_{1} * \ln d ; h=b_{o}+b_{1} * 1 / d+b_{2} * d^{2} ; h\right.$ $\left.=b_{0}+b_{1} * d+b_{2} * d^{2} ; \log h=b_{0}+b_{1} * 1 / d+b_{2} * 1 / I+b_{3} * 1 / D * I ; h=d^{2} /\left(b_{0}+b_{1} * d+b_{2} * d^{2}\right)\right)$ pode ser utilizado. O modelo $\log \mathrm{h}=1,482-2,635 * 1 / \mathrm{d}-0,880 * 1 / \mathrm{I}+3,173 * 1 / \mathrm{D} * \mathrm{I}$ apresenta maior precisão estatística para estimativa dos dados de altura em função do diâmetro e da idade, para povoamentos com $2,4,6$ e 8 anos de idade.

$\mathrm{O}$ volume total com e sem casca foi obtido com base nas equações propostas por Schneider e Hosokawa (1978), a partir dos dados de DAP e de altura do povoamento (Tabela 3).

A partir dos dados do inventário, foi obtida a caracterização do povoamento quanto à distribuição diamétrica. O número de classes foi calculado utilizando-se a fórmula de Sturges descrita por Finger (1992), a qual é expressa por $\mathrm{K}=1+3,3 * \log \mathrm{n}$, em que $\mathrm{n}=$ número de observações e $\mathrm{K}=$ número de classes. Também foi determinado o intervalo de classes com base na fórmula $h=H / K$, em que $H=$ amplitude total (DAP máx - DAP mín), $\mathrm{K}=$ número de classes e $\mathrm{h}=$ intervalo de classe $(\mathrm{cm})$. A figura 1 traz a distribuição do número de árvores por hectare. Observa-se que a maioria das árvores encontra-se nas classes intermediárias. 
Tabela 3. Modelos utilizados para cálculo do volume total com e sem casca para Acacia mearnsii com quatro anos de idade.

Table 3. Models used to calculate the total volume with and without bark for Acacia mearnsii with four years old.

\begin{tabular}{lcc}
\hline \multirow{2}{*}{ Modelos } & \multicolumn{2}{c}{ Coeficientes } \\
\cline { 2 - 3 } & $\mathbf{b}_{\mathbf{0}}$ & $\mathbf{b}_{\mathbf{1}}$ \\
\hline $\log \mathrm{vcc}=\mathrm{b}_{0}+\mathrm{b}_{1} * \log \left(\mathrm{DAP}^{2} \mathrm{~h}\right)$ & $-4,20076$ & 0,9494568 \\
$\log \mathrm{vsc}=\mathrm{b}_{0}+\mathrm{b}_{1} * \log \left(\mathrm{DAP}^{2} \mathrm{~h}\right)$ & $-4,29654$ & 0,9562276 \\
\hline
\end{tabular}

h: altura $(\mathrm{m})$; log: logaritmo de base 10; DAP: diâmetro à altura do peito $(\mathrm{cm})$; vsc: volume total sem casca $\left(\mathrm{m}^{3}\right)$; vcc: volume total com casca $\left(\mathrm{m}^{3}\right)$

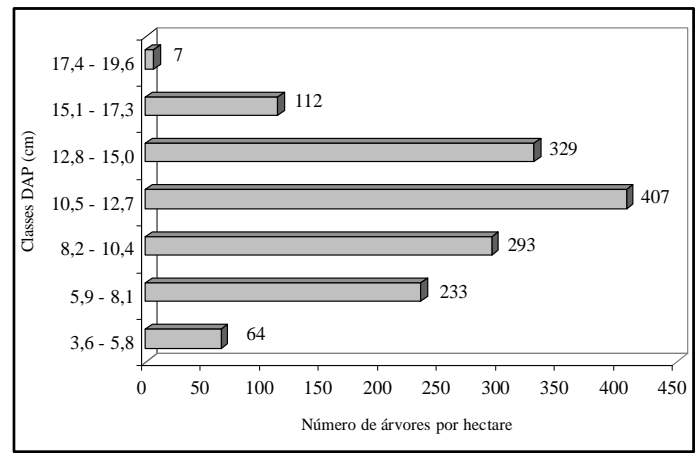

Figura 1. Número de árvores por hectare de Acacia mearnsii distribuídas nos intervalos de classes de DAP.

Figure 1. Number of trees per hectare of Acacia mearnsii distributed in BHD classes intervals.

\section{Teor de micronutrientes nos componentes arbóreos}

$\mathrm{Na}$ tabela 4, constam os teores dos micronutrientes para os diferentes componentes da biomassa das árvores de acácia-negra. Nota-se que as folhas apresentam os maiores teores de B, Cu e Mn. Teores mais altos de $\mathrm{Zn}$ foram encontrados nos galhos mortos. Nas raízes, foram obtidos os maiores teores de Fe. Resultado semelhante foi observado por Hoppe (2003), que associou esse fato a uma possível contaminação da amostra usada para a determinação dos teores de $\mathrm{Fe}$ na raiz, com óxidos de ferro presentes no solo.

Tabela 4. Teores médios de micronutrientes $\left(\mathrm{mg} \cdot \mathrm{kg}^{-1}\right)$ nos diferentes componentes das árvores de Acacia mearnsii.

Table 4. Micronutrients average levels $\left(\mathrm{mg}^{\mathrm{kg}} \mathrm{kg}^{-1}\right)$ in different components of Acacia mearnsii trees.

\begin{tabular}{lccccc}
\hline \multirow{2}{*}{ Componentes } & $\mathbf{B}$ & $\mathbf{C u}$ & $\mathbf{F e}$ & $\mathbf{M n}$ & Zn \\
\cline { 2 - 6 } & & & $\mathbf{m g . \mathbf { k g } ^ { - 1 }}$ & & \\
\hline Folha & $86,49^{1}$ & 14,26 & 137,72 & 86,35 & 18,64 \\
Raiz & 26,63 & 3,28 & 522,50 & 18,70 & 13,64 \\
Madeira & 6,88 & 1,57 & 11,88 & 6,04 & 7,10 \\
Casca & 34,43 & 2,74 & 85,51 & 29,70 & 13,90 \\
Galho morto & 29,52 & 6,49 & 94,20 & 28,70 & 27,55 \\
Galho vivo & 30,06 & 3,73 & 61,60 & 23,40 & 24,81 \\
\hline
\end{tabular}

${ }^{1}$ Teores médios de sete árvores

Em oposição aos altos teores dos micronutrientes encontrados nas folhas, verificou-se que os menores teores foram localizados no componente madeira. A concentração de nutrientes das folhas das árvores é influenciada por diversos fatores, como a idade das árvores, a estação do ano (VAN DER DRIESSCHE, 1984; BELLOTE, 1990), as condições do sítio e a posição em que as folhas estão dispostas na copa (VAN DER DRIESSCHE, 1984).

Para Gonçalves et al. (2000), os maiores teores de nutrientes são encontrados nas partes das plantas mais ativas metabolicamente, como as folhas, e as menores concentrações de micronutrientes são 
encontradas na madeira. Contudo, para a maioria dos nutrientes, é na madeira que se encontram os maiores conteúdos desses nutrientes, simplesmente devido a sua maior massa seca.

Os resultados apresentados por Caldeira (2003) corroboram o que diz a literatura, de que, em geral, as espécies variam no que se refere aos teores de micronutrientes e nas taxas de absorção, bem como na capacidade de reciclagem de nutrientes. Nesse sentido, cabe ressaltar que as necessidades de micronutrientes por parte das espécies são bastante variáveis.

Independentemente da espécie ou da floresta, na grande maioria (CALDEIRA, 2003), os maiores teores de micronutrientes se encontram nas folhas, devido à maior concentração de células vivas presentes nesse componente, que tendem a acumular maiores quantidades de nutrientes, em função dos processos de transpiração e fotossíntese (MARSCHNER, 1997; LAMBERS, CHAPIN; PONS et al., 2000).

\section{Estimativas da quantidade de micronutrientes arbóreos}

As equações ajustadas para estimar os micronutrientes por árvore podem ser observadas na tabela 5. A variável DAP teve um bom ajuste dos dados, expresso pelo alto coeficiente de determinação e pelo baixo erro padrão apresentado pela maioria das equações. Desse modo, é indicada para a retirada de amostras e estimativa de micronutrientes da árvore, por apresentar facilidade de obtenção em campo.

\section{Quantidade de micronutrientes nos componentes da biomassa por hectare}

$\mathrm{O}$ estoque de micronutrientes alocados na biomassa dos diferentes componentes das árvores de acácia-negra é apresentado na tabela 6. Observa-se, mais uma vez, que a maior quantidade está localizada na madeira, exceto o $\mathrm{Fe}$, que apresenta os maiores valores nas raízes, isso provavelmente devido ao teor do elemento nesse componente.

No que se refere ao Fe, Caldeira (2003) observa que na Floresta Ombrófila Mista Montana o teor e o conteúdo de micronutrientes na biomassa radicial apresentou a seguinte ordem: $\mathrm{Fe}>\mathrm{Mn}>\mathrm{Zn}>\mathrm{B}>$ $\mathrm{Cu}$. Essa sequência também foi observada em um Bosque Pluvial de Terras Baixas na Colômbia (RODRIGUES JIMÉNEZ, 1988). Da quantidade total de todos os micronutrientes analisados na biomassa radicial, a contribuição do $\mathrm{Fe}$ foi de aproximadamente de $76,1 \%$ em relação à quantidade total de todos os micronutrientes. Isso, segundo Caldeira (2003), pode ser devido à contaminação pelo solo, motivo pelo qual, em trabalhos com biomassa radicial, as raízes devem ser bem lavadas.

Estudo realizado por Barrichello (2003) em povoamentos de acácia-negra com oito anos de idade, os maiores acúmulos de micronutrientes ocorreram na madeira, exceto para o ferro, que apresentou um maior conteúdo nas raízes. Contudo, para Calil (2003), a madeira de acácia-negra com seis anos de idade apresentou os maiores conteúdos de $\mathrm{B}$ e $\mathrm{Cu}$, enquanto as folhas apresentaram os maiores conteúdos dos micronutrientes Fe e Mn, e os galhos vivos apresentaram os maiores conteúdos de $\mathrm{Zn}$.

Weber (2004), trabalhando com povoamentos de Cryptomeria japonica, constatou que os micronutrientes $\mathrm{B}, \mathrm{Cu}, \mathrm{Mn}$ e $\mathrm{Zn}$ apresentaram a maior concentração nas folhas, exceto o ferro, que apresentou maior teor nos galhos mortos. No entanto, em função da maior proporção da biomassa representada pela madeira, ela apresentou as maiores quantidades dos micronutrientes.

Em seu estudo, Caldeira (2003) comenta que a mesma afirmação feita para o conteúdo de macronutrientes serve para os micronutrientes, ou seja, independentemente da espécie, o conteúdo de micronutrientes na biomassa foi maior na biomassa lenhosa (madeira do fuste + casca + galhos). $\mathrm{O}$ conteúdo médio total de micronutrientes ocorreu na seguinte ordem decrescente na biomassa: galho > madeira > casca > folhas > miscelânea, exceto para $\mathrm{Zn}$ : madeira $>$ galhos $>$ casca $>$ folhas $>$ miscelânea. Para os micronutrientes $\mathrm{B}, \mathrm{Cu}, \mathrm{Fe}, \mathrm{Mn}$ e $\mathrm{Zn}$, o conteúdo total e a proporção deles na biomassa foram maiores nos galhos. Em média, 93,1\% desses micronutrientes estavam acumulados na biomassa lenhosa, que representa $32,3 \%$ da biomassa. Por outro lado, apenas $4 \%$ dos micronutrientes foram acumulados na biomassa não-lenhosa (biomassa de folhas), representando $2,4 \%$ da biomassa total.

As diferenças nos conteúdos de micronutrientes entre os dados do presente estudo com dados de outras espécies podem também ser devidas à heterogeneidade do material quanto ao crescimento, compartimentação dos componentes da biomassa e outras características (CALDEIRA, 2003). Porém, as diferenças dos conteúdos de micronutrientes nos componentes da biomassa de acácia-negra podem ser consequência dos baixos teores dos micronutrientes nos tecidos vegetais e no solo, bem como do fator idade do povoamento. 
Tabela 5. Equações de regressão utilizadas para estimar o estoque de micronutrientes (mg.árvore $\mathrm{e}^{-1}$ ) contidos nos diferentes componentes das árvores de Acacia mearnsii com quatro anos de idade.

Table 5. Regression equations utilized to estimate micronutrients stock (mg.trees ${ }^{-1}$ ) in different components of for four years old Acacia mearnsii trees.

\begin{tabular}{|c|c|c|c|}
\hline Nutriente & Equação & $\mathbf{R}_{\text {aj. }}^{2}$ & $\mathrm{~S}_{\mathrm{yx}}(\%)$ \\
\hline \multicolumn{4}{|c|}{ Raiz } \\
\hline B & $\log \mathrm{y}=-0,27296+2,219367 * \log \mathrm{DAP}$ & 0,88 & 8,9 \\
\hline $\mathrm{Cu}$ & $\log y=-1,56772+2,626420 * \log$ DAP & 0,93 & 13,7 \\
\hline $\mathrm{Fe}$ & $\log y=0,619046+2,580158 * \log$ DAP & 0,79 & 8,6 \\
\hline $\mathrm{Mn}$ & $\log y=-0,54704+2,320777 * \log$ DAP & 0,82 & 12,4 \\
\hline $\mathrm{Zn}$ & $\log y=-1,1335+2,807275 * \log$ DAP & 0,93 & 9,6 \\
\hline \multicolumn{4}{|c|}{ Casca } \\
\hline B & $\log \mathrm{y}=-0,177582+2,253781 * \log \mathrm{DAP}$ & 0,97 & 4,2 \\
\hline $\mathrm{Cu}$ & $\log \mathrm{y}=-1,35239+2,311947 * \log \mathrm{DAP}$ & 0,93 & 13,2 \\
\hline $\mathrm{Fe}$ & $\log \mathrm{y}=0,69345+1,780719 * \log \mathrm{DAP}$ & 0,92 & 4,5 \\
\hline $\mathrm{Mn}$ & $\log y=-0,39405+2,388207 * \log$ DAP & 0,97 & 4,6 \\
\hline $\mathrm{Zn}$ & $\log y=-0,6104+2,281792 * \log$ DAP & 0,96 & 6,1 \\
\hline \multicolumn{4}{|c|}{$\begin{array}{ll}\text { Folha } \\
\end{array}$} \\
\hline $\mathrm{B}$ & $\log \mathrm{y}=-1,384560+3,297924 * \log \mathrm{DAP}$ & 0,89 & 12,2 \\
\hline $\mathrm{Cu}$ & $\log \mathrm{y}=-1,740250+2,878827 * \log \mathrm{DAP}$ & 0,81 & 24,2 \\
\hline $\mathrm{Fe}$ & $\log \mathrm{y}=-1,023230+3,143231 * \log \mathrm{DAP}$ & 0,84 & 13,3 \\
\hline $\mathrm{Mn}$ & $\log y=-1,364290+3,277883 * \log$ DAP & 0,85 & 14,6 \\
\hline $\mathrm{Zn}$ & $\log \mathrm{y}=-1,763430+3,021573 * \log \mathrm{DAP}$ & 0,86 & 19,7 \\
\hline \multicolumn{4}{|c|}{$\begin{array}{rr} & \text { Madeira } \\
\end{array}$} \\
\hline $\mathrm{B}$ & $\log \mathrm{y}=-0,40928+2,530303 * \log \mathrm{DAP}$ & 0,98 & 3,8 \\
\hline $\mathrm{Cu}$ & $\log y=-1,22881+2,705179 * \log$ DAP & 0,98 & 6,0 \\
\hline $\mathrm{Fe}$ & $\log y=-0,94072+3,222329 * \log$ DAP & 0,86 & 11,6 \\
\hline $\mathrm{Mn}$ & $\log y=-0,44508+2,458304 * \log$ DAP & 0,87 & 9,7 \\
\hline $\mathrm{Zn}$ & $\log \mathrm{y}=-0,56409+2,692326 * \log \mathrm{DAP}$ & 0,97 & 4,8 \\
\hline \multicolumn{4}{|c|}{ Galho morto } \\
\hline B & $\log y=-0,47650+2,497829 * \log$ DAP & 0,95 & 5,7 \\
\hline $\mathrm{Cu}$ & $\log y=-0,78794+2,126181 * \log$ DAP & 0,88 & 12,1 \\
\hline $\mathrm{Fe}$ & $\log y=0,074886+2,434286 * \log$ DAP & 0,91 & 6,5 \\
\hline Mn & $\log y=-0,49426+2,497661 * \log$ DAP & 0,94 & 6,5 \\
\hline $\mathrm{Zn}$ & $\log y=-0,36522+2,338432 * \log$ DAP & 0,90 & 8,3 \\
\hline \multicolumn{4}{|c|}{$\begin{array}{ll}\text { Galho vivo } \\
\end{array}$} \\
\hline B & $\log y=-0,44348+2,470468 * \log$ DAP & 0,93 & 6,6 \\
\hline $\mathrm{Cu}$ & $\log y=-0,55661+1,590749 * \log$ DAP & 0,67 & 22,7 \\
\hline $\mathrm{Fe}$ & $\log y=-0,28109+2,613908 * \log$ DAP & 0,91 & 7,1 \\
\hline $\mathrm{Mn}$ & $\log y=-0,81012+2,726324 * \log$ DAP & 0,93 & 7,6 \\
\hline $\mathrm{Zn}$ & $\log y=-0,33029+2,262170 * \log$ DAP & 0,90 & 8,0 \\
\hline
\end{tabular}

Sendo: $\mathrm{R}_{\mathrm{aj}}^{2}$. = coeficiente de determinação; $\mathrm{S}_{\mathrm{yx}}(\%)=$ erro padrão.

Determinação do ponto de amostragem para a quantificação dos micronutrientes no fuste

Por meio do teste de Tukey ao nível de 5\%, não foi verificada diferença significativa entre a altura de amostragem e os teores médios dos micronutrientes ao longo do fuste (Tabela 7). Nesse sentido, Andrae e Krapfenbauer (1983) determinaram que, para Eucalyptus saligna, a altura do ponto de amostragem para o teor dos nutrientes fica em torno de $23 \%$ da altura total. Caldeira (1998) e Barrichello (2003), para Acacia mearnsii com 2,4 e 8 anos, respectivamente, e Weber (2004), para um povoamento de Cryptomeria japonica com 28 anos, utilizaram 50\% da altura total para retirada da amostra do fuste (casca + madeira) para determinação do teor de nutrientes nesses componentes, bem como para determinação do teor de umidade, para o cálculo da biomassa seca. 
Tabela 6. Quantidade de micronutrientes $\left(\mathrm{g}_{\text {.ha }}{ }^{-1}\right)$ encontrada na biomassa, por hectare, nos diferentes componentes das árvores de Acacia mearnsii.

Table 6. Micronutrients quantity $\left(\mathrm{g} \cdot \mathrm{ha}^{-1}\right)$ found in different components biomass, per hectare, of Acacia mearnsii trees.

\begin{tabular}{lcccccc}
\hline \multirow{2}{*}{ Componente } & \multicolumn{5}{c}{ Micronutrientes $\left(\mathbf{g . h a}^{-\mathbf{1}}\right)$} \\
\cline { 2 - 7 } & $\mathrm{B}$ & $\mathrm{Cu}$ & $\mathrm{Fe}$ & $\mathrm{Mn}$ & $\mathrm{Zn}$ & Total \\
\hline Folha & 209,57 & 34,55 & 333,71 & 209,23 & 45,17 & 832,23 \\
Raiz & 216,25 & 26,64 & $4.243,05$ & 151,86 & 110,77 & $4.748,56$ \\
Madeira & 320,73 & 73,19 & 553,82 & 281,57 & 330,99 & $1.560,31$ \\
Casca & 244,19 & 19,43 & 606,48 & 210,65 & 98,59 & $1.179,34$ \\
Exportação de micronutrientes (madeira & 46,0 & 47,0 & 19,0 & 46,0 & 53,0 & - \\
+ casca) em percentagem & 98,93 & 21,75 & 315,71 & 96,19 & 92,33 & 624,91 \\
Galho morto & 159,32 & 19,77 & 326,48 & 124,02 & 131,49 & 761,08 \\
Galho vivo & $1.249,01$ & 195,33 & $6.379,25$ & $1.073,52$ & 809,33 & $9.706,44$ \\
\hline Total & & & & & & \\
\hline
\end{tabular}

Tabela 7. Teores médios de micronutrientes ao longo do fuste para os componentes madeira e casca de Acacia mearnsii com quatro anos de idade.

Table 7. Micronutrients average levels along trunk for wood and bark components of four years old Acacia mearnsii.

\begin{tabular}{|c|c|c|c|c|c|}
\hline \multirow{2}{*}{$\begin{array}{l}\text { \% da altura } \\
\text { total }\end{array}$} & \multicolumn{5}{|c|}{ Nutrientes (mg.kg-1) } \\
\hline & B & $\mathbf{C u}$ & $\mathrm{Fe}$ & Mn & $\mathbf{Z n}$ \\
\hline & \multicolumn{5}{|c|}{ Madeira } \\
\hline $1 \%$ & $6,44 a^{1}$ & $1,62 \mathrm{a}$ & $12,40 \mathrm{a}$ & $5,49 \mathrm{a}$ & $6,12 \mathrm{a}$ \\
\hline $3 \%$ & $5,61 \mathrm{a}$ & $1,51 \mathrm{a}$ & $11,96 \mathrm{a}$ & $5,01 \mathrm{a}$ & $6,55 \mathrm{a}$ \\
\hline $10 \%$ & $5,22 \mathrm{a}$ & $1,41 \mathrm{a}$ & $7,32 \mathrm{a}$ & $5,72 \mathrm{a}$ & $5,65 \mathrm{a}$ \\
\hline $20 \%$ & $6,14 \mathrm{a}$ & $1,27 \mathrm{a}$ & $5,68 \mathrm{a}$ & $4,57 \mathrm{a}$ & $5,17 \mathrm{a}$ \\
\hline $30 \%$ & $5,61 \mathrm{a}$ & $1,47 \mathrm{a}$ & $8,33 \mathrm{a}$ & $5,32 \mathrm{a}$ & $6,10 \mathrm{a}$ \\
\hline $40 \%$ & $6,15 \mathrm{a}$ & $1,46 \mathrm{a}$ & $12,22 \mathrm{a}$ & $5,34 \mathrm{a}$ & $7,63 \mathrm{a}$ \\
\hline $50 \%$ & $6,78 \mathrm{a}$ & $1,71 \mathrm{a}$ & 8,60 a & $4,97 \mathrm{a}$ & $7,14 \mathrm{a}$ \\
\hline $60 \%$ & $6,33 \mathrm{a}$ & $1,18 \mathrm{a}$ & $10,60 \mathrm{a}$ & $5,26 \mathrm{a}$ & $6,58 \mathrm{a}$ \\
\hline $70 \%$ & $6,20 \mathrm{a}$ & $1,64 \mathrm{a}$ & $5,05 \mathrm{a}$ & $5,31 \mathrm{a}$ & $6,67 \mathrm{a}$ \\
\hline $80 \%$ & $7,20 \mathrm{a}$ & $1,56 \mathrm{a}$ & $12,77 \mathrm{a}$ & $6,77 \mathrm{a}$ & $7,11 \mathrm{a}$ \\
\hline \multirow[t]{2}{*}{$90 \%$} & $8,00 \mathrm{a}$ & $1,49 \mathrm{a}$ & $9,08 \mathrm{a}$ & $4,70 \mathrm{a}$ & $7,27 \mathrm{a}$ \\
\hline & \multicolumn{5}{|c|}{ Casca } \\
\hline $1 \%$ & $31,80 \mathrm{a}$ & $3,21 \mathrm{a}$ & $88,47 \mathrm{a}$ & $32,76 \mathrm{a}$ & $13,13 \mathrm{a}$ \\
\hline $3 \%$ & $28,88 \mathrm{a}$ & $2,59 \mathrm{a}$ & $84,49 \mathrm{a}$ & $29,39 \mathrm{a}$ & $12,26 \mathrm{a}$ \\
\hline $10 \%$ & $29,35 \mathrm{a}$ & $2,44 \mathrm{a}$ & $81,45 \mathrm{a}$ & $29,10 \mathrm{a}$ & $13,30 \mathrm{a}$ \\
\hline $20 \%$ & $33,29 \mathrm{a}$ & $2,10 \mathrm{a}$ & $63,98 \mathrm{a}$ & $30,61 \mathrm{a}$ & $12,15 \mathrm{a}$ \\
\hline $30 \%$ & $31,89 \mathrm{a}$ & $2,76 \mathrm{a}$ & $84,78 \mathrm{a}$ & $28,32 \mathrm{a}$ & $12,66 \mathrm{a}$ \\
\hline $40 \%$ & $33,53 \mathrm{a}$ & $2,51 \mathrm{a}$ & $86,46 \mathrm{a}$ & $30,61 \mathrm{a}$ & $12,37 \mathrm{a}$ \\
\hline $50 \%$ & $38,14 \mathrm{a}$ & $2,96 \mathrm{a}$ & $81,61 \mathrm{a}$ & $29,21 \mathrm{a}$ & $13,66 \mathrm{a}$ \\
\hline $60 \%$ & $33,29 \mathrm{a}$ & $2,65 \mathrm{a}$ & $69,47 \mathrm{a}$ & $27,55 \mathrm{a}$ & $14,41 \mathrm{a}$ \\
\hline $70 \%$ & $32,90 \mathrm{a}$ & $2,75 \mathrm{a}$ & $69,02 \mathrm{a}$ & $28,56 \mathrm{a}$ & $14,10 \mathrm{a}$ \\
\hline $80 \%$ & 36,18 a & $2,75 \mathrm{a}$ & $77,97 \mathrm{a}$ & $30,10 \mathrm{a}$ & $11,90 \mathrm{a}$ \\
\hline $90 \%$ & $37,97 \mathrm{a}$ & $2,99 \mathrm{a}$ & $67,27 \mathrm{a}$ & $26,53 \mathrm{a}$ & $15,03 \mathrm{a}$ \\
\hline
\end{tabular}

${ }^{1}$ Teores médios de sete árvores. Médias seguidas pela mesma letra na vertical não diferem pelo Teste de Tukey em 5\% de probabilidade.

As diferenças na altura do ponto de amostragem para o teor dos micronutrientes entre os dados do presente estudo e dados de outras espécies podem também ser devido à espécie, à idade e a características edafoclimáticas, bem como à competição entre indivíduos. 


\section{CONCLUSÕES}

Os teores dos micronutrientes $\mathrm{B}, \mathrm{Cu}, \mathrm{Fe}, \mathrm{Mn}$ e $\mathrm{Zn}$ não apresentaram variação significativa ao longo do fuste para os componentes madeira e casca. Desse modo, a amostragem para quantificação de nutrientes pode ser feita em qualquer altura.

É possível estimar com segurança o estoque de micronutrientes por compartimento das árvores de Acacia mearnsii com o uso de equações alométricas a partir do DAP.

\section{REFERÊNCIAS}

ANDRAE, F. H.; KRAPFENBAUER, A. Estudos da situação da biomassa e de nutrientes de um reflorestamento de quatro anos com Eucalyptus saligna Smith em Santa Maria, RS. In: ANDRAE, F. H.; KRAPFENBAUER, A. (Eds). Pesquisa Austro-Brasileira 1973-1982 sobre Araucaria angustifolia, Podocarpus lambertii e Eucalyptus saligna. Santa Maria: UFSA; Viena: Universitaet fuer Bodenkultur,, 1983, p. 68-85.

BARICHELLO, L. Quantificação da biomassa e dos nutrientes em floresta de Acacia mearnsii De Wild. na região sul do Brasil. 58 f. Dissertação (Mestrado em Engenharia Florestal) - Universidade Federal de Santa Maria, Santa Maria, 2003.

BELLOTE, A. F. J. Suprimento de nutrientes minerais e crescimento de plantações adubadas de Eucaliptus grandis nos cerrados do Estado de São Paulo. $166 \mathrm{f}$. Tese (Doutorado) - Universidade de Fraiburg, Fraiburg, 1990.

CALDEIRA, M. V. W. Determinação de biomassa e nutrientes em uma Floresta Ombrófila Mista Montana em General Carneiro, Paraná. 176 f. Tese (Doutorado em Ciências Florestais) - Setor de Ciências Agrárias, Universidade Federal do Paraná, Curitiba, 2003.

CALDEIRA, M. V. W. Quantificação da biomassa e do conteúdo de nutrientes em diferentes procedências de Acácia-negra (Acacia mearnsii De Wild.). 96 f. Dissertação (Mestrado em Engenharia Florestal) - Universidade Federal de Santa Maria, Santa Maria, 1998.

CALDEIRA, M. V. W.; SCHUMACHER, M. V.; SCHEEREN, L. W.; BARICHELLO, L. R.; WATZLAWICK, L. F. Relação hipsométrica para Acacia mearnsii com diferentes idades. Boletim de Pesquisa Florestal, Colombo, n. 45, p. 57-68, 2002.

CALIL, F. N. Aspectos da ciclagem de nutrientes em um sistema silvopastoril com Acacia mearnsii De Wild., no município de Tupanciretã, RS. 77 f. Dissertação (Mestrado em Engenharia Florestal) Universidade Federal de Santa Maria, Santa Maria, 2003.

CARPANEZZI, A. A. Espécies para recuperação ambiental. In: GALVÃO, A.P.M. Espécies não tradicionais para plantios com finalidades produtivas e ambientais. Colombo: Embrapa Florestas, 1998. p. 43-53.

DRUMOND, M. A.; BARROS, N. F.; SOUZA, A. L.; SILVA, A. F. Distribuição de biomassa e nutrientes em diferentes coberturas florestais e pastagem na região do Medio Rio Doce-MG. Revista Árvore, Viçosa, MG, v. 21, n. 2, p. 187-199, 1997.

EMBRAPA. Cultivo da Acácia-negra. In: Sistemas de Produção, v. 3. (versão eletrônica). Disponível em: <http://sistemasdeproducao.cnptia.embrapa.br/FontesHTML/AcaciaNegra/CultivodaAcaciaNegra/> Acesso em: 23/02/2005.

EMBRAPA. Sistema brasileiro de classificação de solos. 2.ed. Rio de Janeiro: Embrapa Solos, 2006. 306 p.

FINGER, C. A. G. Fundamentos de biometria forestal. Santa Maria: UFSM/CEPEF/FATEC, 1992. 201 p.

GONÇALVES, J. L. M.; STAPE, J. L.; BENEDETTI, V.; FESSEL, V. A. G.; GAVA, J. L. Reflexos do cultivo mínimo e intensivo do solo em sua fertilidade e na nutrição das árvores. In: GONÇALVES, J. L. M.; BENEDETTI, V. (Eds.). Nutrição e fertilização florestal. Piracicaba: ESALQ/USP. p.3-57, 2000. 
HIGA, R. C. V.; DEDECEK, R. A. Acácia-negra. Colombo: EMBRAPA-CNPF, 1999, 2 p. (Instrução Técnica, n. 5).

HOPPE, J. M. Biomassa e nutrientes em Platanus $x$ acerifolia (Aiton) Willd. estabelecido no município de Dom Feliciano-RS. 143 f. Tese (Doutorado em Engenharia Florestal) - Universidade Federal de Santa Maria, Santa Maria, 2003.

INSTITUTO DE PESQUISAS AGRONÔMICAS -IPAGRO. Atlas agroclimático do Estado do Rio Grande do Sul. Porto Alegre, 1989. 3 v.

KIMMINS, J. P. Forest ecology. Canada: Macmillan, 1987. 531 p.

LAMBERS, H.; CHAPIN III, F. S.; PONS, T. L. Plant physiological ecology. New York: SpringerVerlag, 2000. $540 \mathrm{p}$.

MARSCHNER, H. Mineral nutrition of higher pants. 2.ed. San Diego: Academic, 1997. 889 p.

MONTAGNINI, F.; SANCHO, F. Aboveground biomass and nutrient in young plantations of indigenous trees: implications for site nutrient conservation. Journal of Sustainable Forestry, New York, v. 1, n. 4, p. 115-139, 1994.

MORENO, J. A. Clima do Rio Grande do Sul. Porto Alegre: Secretaria da Agricultura, 1961, 42 p.

O'CONNELL, A. M.; SANKARAN, K. V. Organic matter accretion, decomposition and mineralisation. In: NAMBIAR, E. K. S., BROWN, A. G. (Eds.) Management of soil, nutrients and water in tropical plantations forests. Canberra: ACIAR Australia/CSIRO, 1997. p.443-480. (Monograph; n. 43).

OLIVEIRA, H. A. Acácia-negra e tanino no Rio Grande do Sul. Canoas: La Salle, 1968. v. 2.

PEREIRA, J. C.; CALDEIRA, M. V. W.; SCHUMACHER, M. V.; HOPPE, J. M.; SANTOS, E. M. Estimativa do conteúdo de nutrientes em um povoamento de Acacia mearnsii De Wild. no Rio Grande do Sul - Brasil. Revista Árvore, Viçosa, MG, v. 24, n. 2, p. 193-199, 2000.

RESENDE, M. D. V.; HIGA, A. R.; HELLER, J. B.; STEIN, P. P. Parâmetros genéticos e interação genótipo $\mathrm{x}$ ambiente em teste de procedência e progênies de Acácia negra (Acacia mearnsii). Boletim de Pesquisas Florestais, Colombo, n. 24/25, p. 55-65, jan/dez. 1992.

RODRÍGUEZ JIMÉNEZ, L. V. A. Consideraciones sobre la biomasa, composición química y dinámica del bosque pluvial tropical de colinas bajas. Bajo Calima Buenaventura, Colombia. Bogotá: Corporación Nacional de Investigación y Fomento Forestal, 1988. 36 p. (Serie Documentación; n. 16).

SCHENEIDER, P. R.; HOSOKAWA, R. T. Estudo de equações volumétricas para tabelas de volume com e sem casca para Acácia Negra (Acacia mearnsii De Wild.). In: CONGRESSO FLORESTAL BRASILEIRO, 3., 1978, Manaus. Anais... Manaus: Sociedade Brasileira de Silvicultura, 1978. p. 90-95.

SCHNEIDER, P. R.; FLEIG, F. D.; FINGER, C. A. G.; KLEIN. J. E. M. Crescimento da Acácia-negra, Acacia mearnsii De Wild. em diferentes espaçamentos. Ciência Florestal, Santa Maria, v. 10, n. 2, p. 101-112, 2000.

TEDESCO, M. J.; GIANELlO, C.; BISSANI, C. A.; BOHNEN, H.; VOLKWEISS, S. J. Análises de solos, plantas e outros materiais. 2. ed. Porto Alegre: UFRGS, 1995. (Boletim Técnico, n. 5).

VAN DER DRIESSCHE, R. Prediction of mineral status of trees by foliar analysis. The Botanical Review, New York, v. 40, p. 347-394, 1984.

WEBER, C. Biomassa e nutrientes em um povoamento de Cryptomeria japonica (L. F.) D. Don., no município de Encruzilhada do Sul-Rio Grande do Sul. 2004. 36 f. Relatório de Estágio Supervisionado em Engenharia Florestal - Universidade Federal de Santa Maria, Santa Maria, 2004. 